\title{
Bolivia: nuevo sistema electoral presidencial y coordinación política de los partidos
}

\author{
Mario Torrico*
}

\begin{abstract}
Resumen
El presente artículo analiza los incentivos de los sistemas electorales presidenciales en Bolivia. El sistema que estuvo vigente hasta 2005 impulsó la formación de coaliciones de gobierno con el fin de otorgar al Ejecutivo mayoría en el Congreso. Dichas coaliciones fueron perdiendo la confianza del electorado, el cual desde inicios de los noventa buscó opciones políticas alternativas a los partidos más importantes, dando origen a una insatisfacción social con la democracia que desembocaría en la renuncia de Sánchez de Lozada y, posteriormente, el triunfo de Evo Morales. El nuevo sistema electoral para elegir presidente, contenido en la Constitución aprobada en 2009, incrementa las probabilidades de que el Ejecutivo electo no cuente con mayoría legislativa, escenario que en el pasado ha provocado crisis política y la anticipación de elecciones. En un contexto actual más favorable caracterizado por una mayor satisfacción con la democracia y con los partidos, el reto de éstos es tomar decisiones incluyentes, algo inédito en la política boliviana.
\end{abstract}

\begin{abstract}
This article analyzes the incentives generated by presidential electoral systems in Bolivia. The system that was in place until 2005 led to the formation of coalition governments that aimed at giving the Executive the majority in Congress. However, these coalitions gradually lost the electorate confidence, and citizens sought alternative political options to major parties from the early nineties on, giving rise to a social dissatisfaction with democracy. This, in turn, led to the resignation of Sánchez de Lozada and, later, to the triumph of Evo Morales. The new electoral system for electing the president, included in the 2009 Constitution, increases the likelihood that the elected government does not have a majority in Congress. Similar situations in the past led to political crisis and anticipation of elections. In a more favorable context characterized by greater satisfaction with democracy and their parties, the challenge is to take decisions inclusively, something unprecedented in Bolivian politics.

Doctor en Investigación en Ciencias Sociales con mención en Ciencia Política por FLACSO, Sede

México. Profesor-investigador de FLACSO, Sede Méxic
\end{abstract}

Palabras clave: Bolivia, sistemas electorales, relaciones ejecutivo-legislativo, partidos políticos, percepciones políticas, diseño institucional.

Key words: Bolivia, electoral systems, executive-legislative relations, political parties, political perceptions, institutional design.

* Doctor en Investigación en Ciencias Sociales con mención en Ciencia Política por FLACSO, Sede México. Profesor-investigador de FLACSO, Sede México. 
democracia en Bolivia actualmente lleva más de treinta años de vida ininterrumpida, lo que constituye el período más largo de estabilidad política en ese país. En una nación caracterizada históricamente por la continua alternancia entre elecciones generales y golpes de estado, este logro no es menor, como tampoco lo es el hecho de que los resultados electorales en estos años no han sido impugnados por las fuerzas derrotadas o por actores externos al sistema de partidos, lo que otorgó a las autoridades electas la legitimidad necesaria para, al menos, iniciar sus períodos de gobierno $\mathrm{y}$ tomar decisiones.

Las tres décadas que se señalan no han estado exentas de tensiones. En este tiempo han habido momentos en que la preservación de la democracia ha estado comprometida, y para sortearlos se ha tenido que, por un lado, modificar reglas que eran consideradas fundamentales para el sostenimiento democrático y, por otro, abrir el sistema político a actores no formales, admitir nuevas formas de representación y de toma de decisiones. En este proceso han emergido y sucumbido partidos y actores políticos, lo que ha provocado el uso de la violencia por parte del Estado y la respuesta agresiva de grupos organizados. Una constante de la vida política en Bolivia es la combinación de la acción por vías institucionales y por vías extra institucionales e incluso ilegales.

En un país unitario con un sistema presidencialista en el que la elección de las principales autoridades (prefectos, senadores y diputados) dependía del voto otorgado a los candidatos presidenciales, la selección del Presidente de la República ha sido tradicionalmente el elemento central de la lucha política. Más allá de que entre una elección y otra se produjeron hechos y fenómenos que afectaron de manera importante el devenir de la democracia, las reglas para elegir al presidente influyen hasta el día de hoy, en la forma en que se coordinan los partidos y actores políticos, en cómo interactúan y en la manera en que surge y se resuelve el conflicto político.

En el período posterior a las dictaduras militares, Bolivia ha tenido dos tipos de sistema electoral presidencial, ambos de naturaleza mayoritaria. ${ }^{1}$ El sistema de segunda vuelta congresal estuvo vigente por más de veinte años y el de segunda vuelta electoral es de reciente creación y sólo ha sido

1 Se quiere destacar el carácter mayoritario (más del 51\%) en oposición a la posibilidad de elegir a un presidente por una pluralidad (mayoría simple) de votos, como sucede en México 
utilizado en las elecciones presidenciales de finales de 2009. A partir de un análisis comparado de su diseño, el presente trabajo evaluará los resultados del primero y realizará un ejercicio prospectivo sobre los efectos del segundo. Faltando un poco más de un año para que se realicen las próximas elecciones es pertinente ir visualizando los posibles escenarios futuros con base en las experiencias del pasado, especialmente si tomamos en cuenta que la democracia boliviana contemporánea siempre buscó eludir la formación de gobiernos sin mayorías congresales y dadas las nuevas reglas, se abre la posibilidad de que surjan gobiernos de este tipo.

\section{El sistema de segunda vuelta congresal y los incentivos para la coordinación entre partidos}

La Constitución Política de 1967 estableció el método de elección para presidente que estuvo vigente hasta 2009, y consistía en otorgar el triunfo al candidato que hubiera obtenido una mayoría absoluta de votos. En caso de que ninguno llegara a obtener los votos necesarios para ello la presidencia se definiría en una segunda vuelta congresal, en la que participarían los diputados y senadores recientemente electos (en Bolivia la elección presidencial y la legislativa son concurrentes). En 1994 se introdujo una modificación a este sistema de elección, que aunque no alteró su esencia no se puede menospreciar, dicho cambio consistió en restringir la segunda vuelta a los dos candidatos más votados y que los legisladores sólo podían votar por la fórmula de cada partido (la dupla para presidente y vicepresidente). ${ }^{2}$ Anteriormente los tres candidatos presidenciales (y sus respectivos acompañantes vicepresidenciales) con mayor cantidad de votos podían participar en la votación legislativa, y se realizaban dos rondas de votación: una para elegir al presidente y otra para definir el vicepresidente. ${ }^{3}$ Esto último hizo posible que en 1989 accediera a la presidencia quien obtuvo el tercer lugar en las elecciones generales y que su vicepresidente fuera el acompañante

2 Si en las elecciones generales ninguna de las fórmulas para Presidente y Vicepresidente de la República obtuviera la mayoría absoluta de sufragios válidos, el Congreso elegirá por mayoría absoluta de votos válidos, en votación oral y nominal, entre las dos fórmulas que hubieran obtenido el mayor número de sufragios válidos" (Artículo 90 de la CPE de 1967, modificado por Ley No1585 del 12 de agosto de 1994).

3 Si ninguno de los candidatos para la Presidencia o la Vicepresidencia de la República obtuviese mayoría absoluta de votos, el Congreso tomará a tres de los que hubiesen obtenido el mayor número para uno u otro cargo y de entre ellos hará la elección" (Artículo 90 de la CPE de 1967) 
de fórmula de otro candidato presidencial (Jaime Paz Zamora del MIR y Luis Ossio de ADN, respectivamente).

Entre 1980 y 2002 ningún candidato presidencial obtuvo una mayoría absoluta de votos en las elecciones generales, por lo que todos los presidentes fueron electos en la segunda vuelta congresal establecida constitucionalmente. Este hecho acerca el presidencialismo boliviano de ese período a un régimen de tipo parlamentario, en el que líder del gobierno es elegido por el poder legislativo. Sin embargo, una vez electos el presidente y el vicepresidente, su permanencia no dependía de preservar la confianza del congreso, rasgo típico de un régimen presidencial en el que ambos poderes tienen etapas de mandatos fijos e independientes respecto al apoyo de uno en el otro (Linz, 1994). La confluencia de ambos aspectos (elección legislativa del ejecutivo e independencia de períodos de mandatos) provocó que el sistema político boliviano fuera caracterizado como un presidencialismo parlamentarizado (Mayorga, 2000). ${ }^{4}$

Más allá de señalar las características del sistema electoral presidencial boliviano vigente hasta la década pasada, en este documento interesa analizar los efectos que tuvo en la coordinación de los actores y partidos a nivel electoral, en su interacción política en lapsos interelectorales y en el surgimiento y resolución de conflictos. Por ello es importante señalar, en primer lugar, los incentivos que proporcionaba a los actores y a los partidos políticos.

La Ciencia Política ha abordado ampliamente los efectos de los sistemas electorales en los sistemas de partidos (Duverger, 1961; Rae, 1967; Taagepera y Shugart, 1989; Cox, 1997; Reynolds et al., 2006), y aunque son generalmente pensados para la elección de legisladores (debido a que los países tradicionalmente democráticos son en su mayoría parlamentarios, y a que el distrito electoral en una elección presidencial es de un único miembro, por lo que la aplicación en él de una fórmula electoral proporcional no tendría sentido), sus conclusiones resultan muy útiles para el análisis de los sistemas electorales presidenciales.

Para la elección de presidente sólo se puede aplicar alguna variante de la familia de las fórmulas electorales mayoritarias (pluralidad o mayoría simple y mayoría absoluta con segunda vuelta o balotaje), y de esto dependerá en buena medida los incentivos para que los partidos construyan coaliciones pre o poselectorales y para que los votantes decidan emitir un

4 En América Latina este sistema existió también en Chile (antes de la dictadura de Pinochet), El Salvador (hasta 1983) y Guatemala (hasta 1985). 
voto sincero o estratégico. Según lo que nos indican los postulados teóricos y los hallazgos empíricos politológicos, en un sistema de mayoría simple el número efectivo de partidos tenderá a dos debido a que los electores concentran su voto en las dos opciones más viables de obtener la victoria, motivo por el que los partidos pequeños tienen incentivos a unirse a alguna de las candidaturas de partidos grandes, ya que de lo contrario no tendrían la posibilidad de conseguir espacios de poder. En consecuencia se produce el surgimiento de coaliciones preelectorales.

En sistemas de mayoría absoluta con posibilidad de segunda vuelta el número de partidos aumenta debido a que los votantes tienen la posibilidad de ejercer en la primera vuelta un voto sincero de acuerdo a sus preferencias electorales, por lo que los partidos pequeños tienen incentivos a presentarse con candidato propio para que en una eventual segunda ronda, negociar su apoyo con alguno de los partidos que accedieran a esta instancia. Este apoyo además podría dar lugar a la creación de una coalición de gobierno, situación muy benéfica para los partidos que inicialmente no tenían posibilidad de disputar realistamente la presidencia con los partidos con mayor apoyo electoral.

Un supuesto de los estudios sobre sistemas electorales mayoritarios es que en la segunda ronda también se efectúa un voto ciudadano, situación que no aplica para el análisis del caso boliviano. La segunda vuelta congresal introduce mayor complejidad al análisis de los efectos del sistema electoral presidencial, ya que se produce una delegación del elector a favor de los legisladores para que éstos elijan al presidente (y antes de 1994, también al vicepresidente). Estos legisladores en Bolivia no habían sido elegidos directamente por los ciudadanos en la primera ronda, ya que el método de elección del congreso era por medio de listas cerradas y bloqueadas. ${ }^{5} \mathrm{Ni}$ siquiera había la posibilidad de diferenciar el voto por el candidato presidencial del voto por el partido en el congreso, lo que significa que el Poder Legislativo se conformaba a partir de la votación por los candidatos presidenciales (los electores sólo podían emitir un voto en una única boleta). ¿Qué incentivos a partidos y a electores genera este tipo de diseño institucional?

Cox (1997) nos indica que en una elección el número de candidaturas tenderá a ser igual a $\mathrm{m}+1$, donde $\mathrm{m}$ es el tamaño del distrito. En un sistema electoral presidencial de mayoría simple el número de partidos tendería a

5 En la reforma de 1994 se implementó un sistema electoral mixto, aspecto que se analizará más adelante 
dos (como ya lo había señalado mucho tiempo antes Duverger). La novedad que ofrece Cox se refiere a los sistemas de doble vuelta, donde la regla anterior se mantiene pero m es igual al número de contendientes que puede pasar a la segunda ronda. En este sentido antes de 1994 en Bolivia el número de candidaturas presidenciales debería tender a cuatro, y entre ese año y 2005 debería estar alrededor de tres. Para examinar si esto se ajusta a lo que sucedió recurriremos a analizar el Número Efectivo de Partidos (NEP).

Cuadro 1. Número efectivo de partidos, 1980 - 2005

\begin{tabular}{|c|c|c|c|c|c|c|c|}
\hline Año & 1980 & 1985 & 1989 & 1993 & 1997 & 2002 & 2005 \\
\hline NEP & 4.35 & 4.58 & 5.02 & 5.15 & 5.91 & 5.77 & 2.62 \\
\hline
\end{tabular}

Entre 1980 y 1993, período en el cual estuvo vigente un sistema electoral presidencial que habilitaba a los tres primeros lugares de la elección a disputar la presidencia en una segunda ronda congresal, el NEP siempre fue superior a cuatro; es más este indicador fue aumentando en el tiempo, incluso después de que se estableció que la segunda vuelta se realizaría sólo entre los dos primeros lugares. Ello revela que lo señalado por Cox no se ajusta a lo que ocurrió en Bolivia. Aunque resulta cierto que un sistema de mayoría absoluta con doble vuelta indujo a la formación de multipartidismo (conforme al planteamiento de Duverger), la pregunta relevante es por qué se generó un sistema de partidos altamente fragmentado.

El NEP es un indicador útil, aunque suele ser criticado debido a que en sus valores influye cuántos partidos participaron en la contienda electoral y la distribución de votos entre ellos (Negretto, 2006). Por ello en el siguiente cuadro se exhibe el Número de Partidos Participantes de la elección (NPP) y el porcentaje de votos obtenido por el ganador. Esta información complementa la presentada en el cuadro 1 para un análisis más exhaustivo.

\section{Cuadro 2. NPP y porcentaje de votos obtenido por el ganador de elecciones} presidenciales, 1980-2005

\begin{tabular}{|c|c|c|c|c|c|c|c|}
\hline Año & 1980 & 1985 & 1989 & 1993 & 1997 & 2002 & 2005 \\
\hline NPP & 13 & 18 & 10 & 14 & 10 & 11 & 8 \\
\hline $\begin{array}{c}\text { \% del } \\
\text { ganador }\end{array}$ & $38.74 \%$ & $32.83 \%$ & $25.64 \%$ & $33.84 \%$ & $22.30 \%$ & $22.50 \%$ & $53.72 \%$ \\
\hline
\end{tabular}

Fuente: Elaboración propia en base a datos de Nohlen (2005) y OEP (2012) 
Aunque el NPP es fluctuante, a partir de 1985 muestra una tendencia descendente en el tiempo, no obstante lo cual el número de candidatos presidenciales nunca dejó de ser muy alto. Sin embargo, lo más destacado es que ninguna candidatura antes de 2005 obtuvo siquiera un cuarenta por ciento de los votos, lo que muestra que las preferencias electorales de los votantes han estado altamente fragmentadas. Por ejemplo en $1997 \mathrm{hu}-$ bo una diferencia de seis puntos porcentuales entre el primer y el quinto lugar, misma diferencia que se observó en 2002 entre el primer y el cuarto partido más votado. Una primera conclusión que se extrae de ello es que la reforma electoral de 1994 no tuvo efectos en disminuir la fragmentación de votos y en reducir la cantidad de candidatos presidenciales. Esto significa que a pesar de la reforma, los partidos políticos no tenían incentivos para coordinarse estableciendo alianzas preelectorales. La apuesta racional, incluso para quienes no tenían la menor posibilidad de ganar las elecciones presidenciales, era presentarse a ellas con candidato propio para intentar obtener algún nivel de representación parlamentaria y negociar su apoyo posteriormente. Por el lado de los electores los incentivos para emitir un voto sincero se potencializaron dado que, ante la expectativa -cumplida elección tras elección- de que los partidos se verían obligados a conformar coaliciones de gobierno, buena parte de los votantes trataba de impulsar que su partido de preferencia ingresara a las mismas. En consecuencia, dado que entre 1980 y 2002 se mantuvieron constantes la fragmentación electoral y los incentivos electorales de los partidos, debemos volcar la mirada a un aspecto de diseño institucional que también se mantuvo constante luego de la reforma de 1994: la segunda ronda congresal.

En sistemas parlamentarios cuando ningún partido obtiene mayoría en el congreso, usualmente es necesario formar coaliciones para nombrar al nuevo gobierno. Esta coalición de partidos se replica en el ejecutivo y en el legislativo, lo que hace que el primero pueda echar a andar su programa de gobierno con el apoyo del segundo (aspecto que era enfatizado por Linz como muy favorable para el sostenimiento de la democracia). En Bolivia, la segunda vuelta congresal, con independencia del número de candidaturas que podían participar en ella, generaba incentivos para que los partidos buscaran coordinarse poselectoralmente, ya que los apoyos en esta instancia se convertían posteriormente en coaliciones de gobierno. ${ }^{6}$

6 Por supuesto que estas decisiones de formar coaliciones de gobierno eran presentadas al público, en la búsqueda de su legitimidad, no sólo como necesarias sino también como indispensables para asegurar la democracia y la gobernabilidad. Así, la coalición entre el Movimiento Nacionalista Revolucionario (MNR) y Acción Democrática Nacionalista (ADN) que gobernó 
La posibilidad de que partidos con un nivel de votación del $5 \%$ pudieran ser parte del gobierno -como ocurrió con MBL y UCS en 2002- provocó que el número de candidaturas fuera muy alto, como se vio en el cuadro 2.

Existen dos elementos adicionales a tomar en cuenta: el umbral electoral y el sistema electoral para elegir legisladores. El umbral en Bolivia se ha caracterizado por ser muy bajo (dos\% antes de la reforma de 1994 y $3 \%$ después de ella), y si los partidos obtenían un nivel de votación menor no perdían el registro automáticamente, se les daba la oportunidad para continuar participando en elecciones posteriores, de resarcir a la Corte Nacional Electoral su fracción del costo de impresión de la boleta electoral. Ello impedía reducir el número de partidos políticos, a pesar de que en todas las elecciones desde 1980 al menos cuatro partidos obtuvieron menor votación que la establecida. El segundo elemento a tomar en cuenta es el sistema electoral para conformar el congreso, el cual era de representación proporcional con lista cerrada y voto único para el elector, lo que provocaba que la distribución de legisladores electos fuera muy cercana a los resultados de la elección presidencial. Si a esto agregamos que la fragmentación de preferencias de los votantes era alta, el resultado es que prácticamente resultaba imposible que algún partido obtuviera mayoría absoluta en el legislativo. En consecuencia, la formación de coaliciones a partir de las cuáles se elegía presidente y luego se gobernaba, era un efecto de la interacción entre el diseño electoral presidencial, el diseño electoral legislativo y la fragmentación de preferencias de los electores.

Con un sistema electoral presidencial de doble ronda congresal, con unas preferencias políticas fragmentadas e incentivadas a manifestarse sinceramente -y no estratégicamente-en el voto, con un umbral muy bajo y con un sistema electoral para el legislativo que impedía la formación de mayorías a menos que un candidato presidencial obtuviera dicha mayoría en votos, no se generaron tendencias centrípetas en el sistema de partidos. Los incentivos por negociar en la segunda vuelta en el congreso los votos de los legisladores preservaron la fragmentación del sistema de partidos. La importancia de las coaliciones poselectorales se aprecia al considerar que los gobiernos que carecieron de ellas tuvieron que llamar a elecciones

entre 1985 y 1989 fue bautizada por ellos mismos como Pacto por la Democracia; la conformada por el Movimiento de Izquierda Revolucionaria (MIR), ADN y Conciencia de Patria (CONDEPA), que tuvo el poder entre 1989 y 1993, fue denominada Acuerdo Patriótico; la que pactaron el MNR, Unidad Cívica Solidaridad (UCS) y el Movimiento Bolivia Libre (MBL) para gobernar entre 1993 y 1997 fue llamada Pacto por la Gobernabilidad; y la que formaron ADN, MIR, UCS y Nueva Fuerza Republicana (NFR) entre 1997 y 2002 se llamó Compromiso por Bolivia. 
anticipadas (Siles en 1985 y Mesa en 2005). Es así que todos los presidentes electos entre 1985 y 2002 formaron coaliciones producto de las negociaciones con otros partidos para poder vencer en la segunda vuelta congresal, práctica que fue conocida peyorativamente como Democracia Pactada.

Cuadro 3. Gobiernos y coaliciones políticas, 1985-2003

\begin{tabular}{|c|c|c|c|c|c|}
\hline $\begin{array}{c}\text { Periodo de } \\
\text { gobierno }\end{array}$ & 1985-1989 & 1989-1993 & $1993-1997$ & $1997-2002$ & $2002-2003$ \\
\hline $\begin{array}{c}\text { Partido del Presi- } \\
\text { dente }\end{array}$ & MNR & MIR & MNR & ADN & MNR \\
\hline $\begin{array}{c}\text { Partidos en la } \\
\text { coalición } \\
\text { de gobierno }\end{array}$ & ADN & ADN & UCS, MBL & $\begin{array}{c}\text { MIR, CON- } \\
\text { DEPA, } \\
\text { UCS, NFR }\end{array}$ & $\begin{array}{c}\text { MIR, UCS, } \\
\text { NFR }\end{array}$ \\
\hline
\end{tabular}

Con todo lo anterior, se puede señalar que las reglas electorales proporcionaban incentivos para que los partidos políticos bolivianos se coordinaran en coaliciones poselectorales que le daban sustento al gobierno a través de la mayoría legislativa con que contaba. Preelectoralmente era racional para los partidos no establecer alianzas para conseguir, dado el bajo umbral electoral y lo poco costoso que resultaba no alcanzarlo, al menos un representante en el congreso que otorgara poder de negociación en la segunda ronda. Ello mantuvo un sistema de partidos altamente fragmentado. Además debido al sistema electoral de representación proporcional con lista cerrada, existía una gran disciplina partidista de los legisladores y las negociaciones políticas se restringían a las cúpulas partidistas. Este esquema le dio estabilidad a la democracia boliviana durante dos décadas, misma que se caracterizaba por la presencia de un Ejecutivo dominante y de un Legislativo subordinado. ${ }^{7}$ ¿Qué cambió para que todo esto se venga abajo en la década pasada?

El cuadro 3 muestra que entre 1985 y 2002 existieron tres partidos dominantes en el sistema político: MNR, ADN y MIR. Todos ellos ocuparon en algún momento la presidencia y en todas las coaliciones de gobierno al menos uno de ellos estuvo presente. Sin embargo, a partir de 1993 se observa el ingreso de otros partidos a las coaliciones, varios de los cuales eran de reciente creación. Ello significa que nuevos partidos (como CONDEPA, UCS Y NFR) lograron buenos resultados electorales en un corto tiempo, ¿qué nos señala ésto?

7 Sobre las relaciones Ejecutivo-Legislativo en América Latina, véase Cox y Morgenstern (2002). 
Cuadro 4. Distribución de votos en procesos electorales

\begin{tabular}{|c|c|c|c|c|c|}
\hline Año de elección & 1985 & 1989 & 1993 & 1997 & 2002 \\
\hline MNR+ADN+MIR & $73.37 \%$ & $72.64 \%$ & $53.87 \%$ & $57.30 \%$ & $42.20 \%$ \\
\hline Otros partidos & $26.63 \%$ & $27.36 \%$ & $46.13 \%$ & $42.70 \%$ & $57.80 \%$ \\
\hline
\end{tabular}

Fuente: Elaboración propia en base a datos de OEP (2012)

En 1985 y 1989 casi 3/4 de los votos fueron para MNR, ADN o MIR. Sin embargo, a partir de 1993 se evidencia un declive en las preferencias por estos partidos y el aumento de la votación de otros, en especial de aquellos liderados por personas provenientes del ámbito empresarial (Carlos Palenque de CONDEPA y Max Fernández de UCS) que enarbolaban un discurso de oposición al "sistema de partidos tradicional" y exhibían prácticas que podrían señalarse como populistas (ambos construían obras en barrios periféricos de las ciudades y en pueblos cercanos a las mismas). El relativo éxito electoral de estos nuevos liderazgos señala la tendencia de que los votantes buscaban alternativas a los tres partidos más importantes y que el sistema de partidos estaba sufriendo un desgaste considerable.

Producto de ello, y para aminorar este desgaste, la reforma electoral de 1994 modificó el sistema de elección de legisladores implementando un sistema electoral mixto, en el que la mitad de la cámara baja se conformaba como antes, es decir, mediante listas cerradas, y la otra mitad por votación directa a candidatos a diputados en distritos uninominales. ${ }^{8} \mathrm{Sin}$ embargo, se mantuvo la proporcionalidad de la representación legislativa de los partidos, mediante un ajuste proporcional a la votación obtenida por el candidato presidencial se compensaba la desproporcionalidad generada por la elección de diputados uninominales. ${ }^{9}$

La reforma electoral de 1994, en lo que se refiere al método de elección del congreso, fue motivada por el cambio de las preferencias electorales (que comenzaban a dejar de estar centradas alrededor de MNR, ADN y MIR) y buscaba darle mayor legitimidad al sistema político, por lo que hubo un amplio consenso de los partidos en su aprobación (Buquet, 2007).

Esto significa que a pesar de la fragmentación del voto, los electores empezaron a coordinarse buscando opciones políticas distintas a los partidos tradicionales, lo que motivó la modificación de las reglas electorales

8 Este sistema fue originalmente formulado en Alemania en la década del cincuenta del siglo XX

9 Una explicación del funcionamiento de los sistemas electorales mixtos se encuentra en Shugart y Wattenberg (2001) 
que se señala. No obstante, la principal característica del sistema, la segunda vuelta congresal para elegir presidente, se mantuvo, lo que propició que los nuevos partidos se incorporaran a las coaliciones de gobierno. ¿Por qué este esquema no perduró?

Coaliciones de gobierno con mayor cantidad de partidos enfrentan desafíos que antes eran menores, por ejemplo, cómo prevenir la corrupción en instituciones gubernamentales controladas por distintos partidos o para los electores, a quién asignar la responsabilidad por errores en la gestión pública. La coordinación al interior de la coalición se vuelve mucho más compleja e incluso imposible, generando serios problemas de eficacia lo cual agrava aún más los problemas de legitimidad. Al respecto, la Ciencia Política ha estudiado ampliamente los problemas de acción colectiva que se generan cuando no se puede identificar a algún miembro de un grupo que sigue una estrategia no cooperativa buscando explotar el esfuerzo de los demás (Olson, 1991) y también lo complejo que resulta la toma de decisiones colectiva (Arrow, 1963; Riker, 1982). Con base en esa literatura podemos entender que en la medida en que el número de partidos al interior de las coaliciones de gobierno aumentaba (como ocurrió a partir de 1993) también se incrementaban los incentivos de cada uno de ellos por tomar ventajas particulares del poder, sean éstas legales o ilegales (por ejemplo, mediante el nombramiento de correligionarios en la administración pública o recurriendo a prácticas de corrupción) ya que no le era fácil al electorado identificar al partido responsable de las mismas y en consecuencia, ejercer un castigo electoral, lo que llevó al desprestigio de todo el sistema de partidos. Por otro lado, un mayor número de miembros en la coalición hacía más difícil para el ejecutivo tomar decisiones aceptables por todos. Esta situación es paradójica ya que en la búsqueda por establecer coaliciones de gobierno que aseguraran al presidente la mayoría congresal para aprobar sus propuestas, fue la situación que después obstaculizó la toma de decisiones, problema que se agudizó durante la presidencia de Bánzer.

En el gobierno de Hugo Bánzer (1997-2002) el descontrol gubernamental y el desconcierto para enfrentar los conflictos sociales fueron muy grandes, al mismo tiempo estallaron escándalos de corrupción que redujeron al mínimo la confianza en los partidos (véase la gráfica 1). Los esfuerzos por hacer funcionar la llamada megacoalición ${ }^{10}$ fueron en vano, y el ejecutivo

10 Este término fue acuñado por los medios de comunicación para resaltar el hecho de que la coalición de partidos que llevó a Bánzer a la presidencia era la más numerosa en la historia democrática boliviana. 
no pudo hacer nada para evitar que en el año 2000 la presión popular movilizada por grupos sociales organizados obligara a rescindir un contrato de privatización del servicio de agua con una empresa transnacional en la ciudad de Cochabamba. Poca legitimidad del sistema de partidos, preferencias electorales en creciente búsqueda de partidos con nuevas opciones frente a los existentes, movilización social y problemas de eficacia de los gobiernos llevaron a niveles muy bajos de satisfacción con la democracia (véase la gráfica 2) que no podía sino tener un desenlace violento, como el que caracterizó el período 2003-2005. ¿Cómo explicar que con las reglas electorales descritas hasta ahora un partido político hubiera podido conseguir la mayoría absoluta de votos y llegar al gobierno sin necesidad de una segunda ronda congresal que lo obligara a establecer una coalición con otros partidos?

\section{Cuando el contexto cambia, las reglas también cambian}

En 2002 el Movimiento al Socialismo (MAS) se benefició del voto de una porción del electorado que desde 1993 buscaba alguna alternativa a los partidos tradicionales. Como UCS y CONDEPA habían formado parte de gobiernos anteriores, ya no eran vistos como alternativas a los mismos e incluso su votación se desplomó. En ese contexto el MAS, que había obtenido el segundo lugar en las elecciones, enfrentaba la decisión de formar coalición con otros partidos para intentar obtener la presidencia en la segunda ronda congresal, acción que lo incorporaría a la democracia pactada. La experiencia de UCS y CONDEPA pudo haber incidido en la decisión que tomó su líder, Evo Morales, de no negociar el apoyo de otros partidos y mantenerse al margen de las coaliciones. También pudo ocurrir que en el cálculo político del MAS no había nada que se pudiera hacer para asegurar el suficiente apoyo que los llevara al gobierno. ${ }^{11}$ Naturalmente, la votación en el congreso favoreció a su contendiente, Gonzalo Sánchez de

11 Frente al sorprendente segundo lugar del MAS, casi de inmediato se empezaron a pronunciar políticos y empresarios sobre la necesidad de que los demás partidos garantizaran la estabilidad de la democracia a través de una actitud responsable que, por sobre sus diferencias, los llevara a conformar una coalición de gobierno en torno al MNR. Unos días después Jaime Paz Zamora, ex presidente de la República y líder del MIR, que había tenido una relación de confrontación con Sánchez de Lozada por muchos años, al anunciar su incorporación al gobierno que encabezaría este último mencionó: “¿Qué difícil es amar a Bolivia!”. La continuidad del sistema político parecía ser prioritaria. 
Lozada, quien con el voto de los representantes de varios partidos accedió a su segundo período presidencial (el primero fue entre 1993 y 1997).

A partir de 2002 se evidenció que el sistema político no podía procesar las demandas de un partido que no se movía solamente en el ámbito formal-institucional, sino que además contaba con una gran capacidad de movilización social y de generación de violencia en calles y carreteras. Finalmente a pesar de contar con el apoyo de más del setenta por ciento del congreso, el gobierno de Sánchez de Lozada cayó en octubre de 2003. El contex to había cambiado y el golpe político vino desde fuera del sistema de partidos, pero todo se originó en el cambio paulatino de las preferencia electorales desde inicios de la década de los años noventa y la incapacidad de los partidos para dar respuesta a esta búsqueda de los electores por nuevas opciones políticas, fue lo que repercutió en la legitimidad de la democracia misma y en que los gobiernos pudieran gobernar con eficacia por las cada vez más numerosas coaliciones.

El período que transcurre entre 2003 y 2005 fue de amplia inestabilidad política y culminó con la convocatoria a elecciones anticipadas, en las cuales Evo Morales obtuvo un 53.7\% de los votos, suficientes para llevarlo directamente a la presidencia sin pasar por la votación del congreso. $\mathrm{Si}$ observamos nuevamente los cuadros 1 y 2 , podemos ver que esa elección exhibe el NEP más bajo de la historia democrática boliviana contemporánea. No obstante, el NPP es aún elevado (al menos si lo comparamos con lo que normalmente sucede en otras democracias), aunque ciertamente en 2005 se registra la menor cantidad de candidatos presidenciales desde 1980. ¿Qué ocurrió?

En 2005 estaba presente la segunda ronda congresal, lo que nuevamente y a pesar de todo el conflicto político y social que se vivió en los años previos, brindó incentivos para que se presentaran muchos partidos a las elecciones. Todos ellos buscaban alguna representación legislativa que les permitiera negociar su apoyo en la segunda vuelta. Además las encuestas no mostraban a un ganador por mayoría absoluta en la primera ronda y tan sólo le otorgaban una ventaja de algunos puntos a Evo Morales por encima de su competidor más cercano, Jorge Quiroga (quien había sido vicepresidente de Bánzer y luego, cuando éste murió, presidente por un año) de Poder Democrático y Social (PODEMOS), que se formó a partir de personajes políticos que anteriormente habían formado parte de ADN y del MIR.

El triunfo del Movimiento al Socialismo en primera ronda fue sorpresivo, y se debió en buena medida a que los electores de áreas rurales incrementaron notablemente su participación (el aparato de movilización 
del sector cocalero tuvo mucho que ver en esto), ${ }^{12}$ aunque también debe resaltarse que los electores que buscaban desde hacía tiempo una opción política diferente a los tres partidos más importantes de las dos décadas previas habían encontrado no sólo atractivo al MAS, sino -lo que es más importante- creíble, debido al antecedente de 2002. Es interesante preguntarse si Evo Morales habría buscado establecer alguna coalición de gobierno para llegar a la presidencia si no hubiera obtenido la mayoría absoluta. Lo más probable es que sí, aunque nunca lo sabremos.

El triunfo de Evo Morales supuso el derrumbe del sistema de partidos como se había conocido hasta entonces. El nuevo gobierno hizo lo que toda coalición ascendente que tenía legitimidad llevara a cabo: una reforma que le permitiera tomar decisiones con eficacia (Buquet, 2007). Aunque el conjunto de reformas que impulsó el MAS es muy amplio, para los propósitos de este artículo sólo interesan las que tienen que ver con el método de elección presidencial.

La Nueva Constitución Política, aprobada por referéndum en 2009 y que emergió de la Asamblea Constituyente de 2008, establece un sistema electoral en el que si ningún candidato presidencial obtiene la mayoría absoluta de votos en la primera vuelta, se realiza una segunda votación ciudadana entre los dos más votados, es decir, se prescinde de la segunda ronda congresal (lo que elimina el aspecto parlamentario del presidencialismo boliviano). Además, se introdujo la posibilidad de reelección inmediata para el presidente. Estas modificaciones beneficiaron al MAS en 2009, que logró la reelección de Evo Morales con un porcentaje de $64.2 \%$, mayor a la votación obtenida en 2005. ¿Cómo se reflejan todos los cambios descritos en la percepción ciudadana? Y lo más importante, ¿qué efectos a mediano plazo se pueden esperar de los cambios institucionales efectuados dado el nuevo contexto político?

\section{Percepciones sobre la democracia}

Bolivia a lo largo de toda su historia ha vivido en permanente crisis política, prueba de ello es el número de golpes de estado que se han producido desde su independencia: 193. Cuando el período de gobiernos militares finalizó en 1982 era prioritaria la estabilidad democrática, y ciertamente los pactos y coaliciones entre partidos ayudaron a alcanzar este fin. Si los

$12 \mathrm{Al}$ respecto, véase el documental Cocalero. 
principales actores políticos tenían posibilidades de insertarse en el poder y beneficiarse de él, las tentaciones por embarcarse en aventuras golpistas tendían a desaparecer. El problema fue que estas prácticas fueron las que deslegitimaron a los partidos y a la propia democracia ante la población. Lamentablemente no tenemos datos sobre percepción política previos a finales de los años noventa, por lo que no se puede observar la tendencia de las opiniones en torno a la democracia y sus actores en todo el período democrático contemporáneo. No obstante, las gráficas siguientes ofrecen una buena imagen de la opinión ciudadana al respecto en los años en que se desarrollaron elecciones presidenciales, antes y después del primer triunfo de Evo Morales. Los datos sobre Bolivia se comparan con los de América Latina en conjunto con el fin de tener una referencia que ayude en el análisis, y concluyen en 2009 año en que dicho líder fue reelecto y último período para el que existen datos disponibles abiertos.

Gráfica 1. Confianza en los Partidos Políticos en Bolivia y América Latina (\%)*

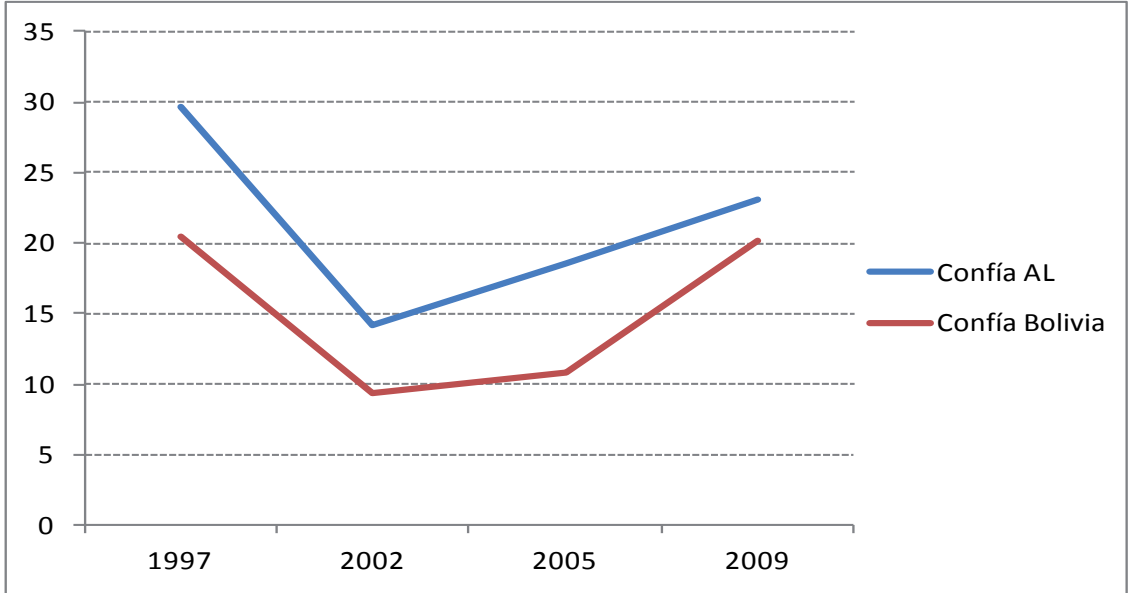

* La categoría "Confía" es la agregación de las respuestas Mucha confianza y Algo de confianza en los par tidos políticos. Las otras posibilidades de respuesta para los encuestados fueron Poca Confianza y Ninguna confianza.

Fuente: Corporación Latinobarómetro (1998), (2003), (2006) y (2010). 


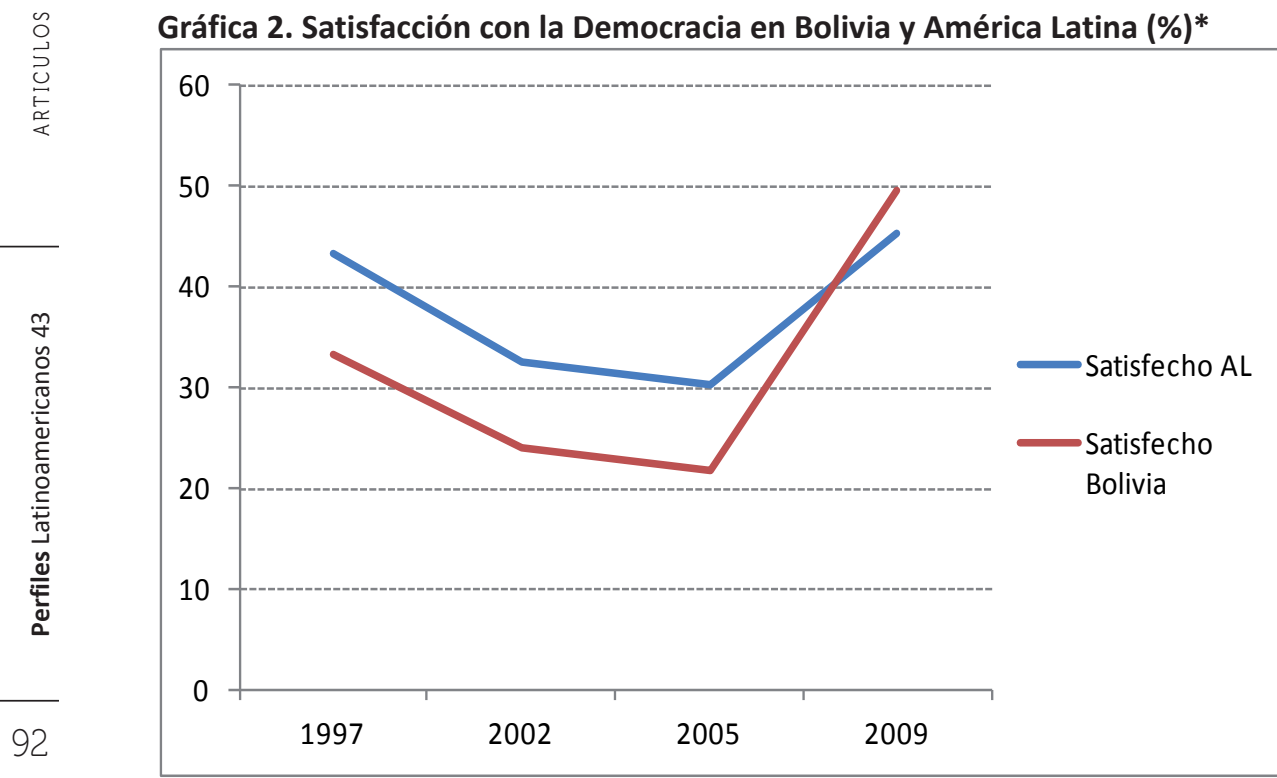

* La categoría "Satisfecho" es la agregación de las respuestas Muy satisfecho y Más bien satisfecho con la democracia. Las otras posibilidades de respuesta para los encuestados fueron No muy satisfecho y Nada satisfecho. Fuente: Corporación Latinobarómetro (1998), (2003), (2006) y (2010).

\section{Gráfica 3. Percepción de aumento de la corrupción*}

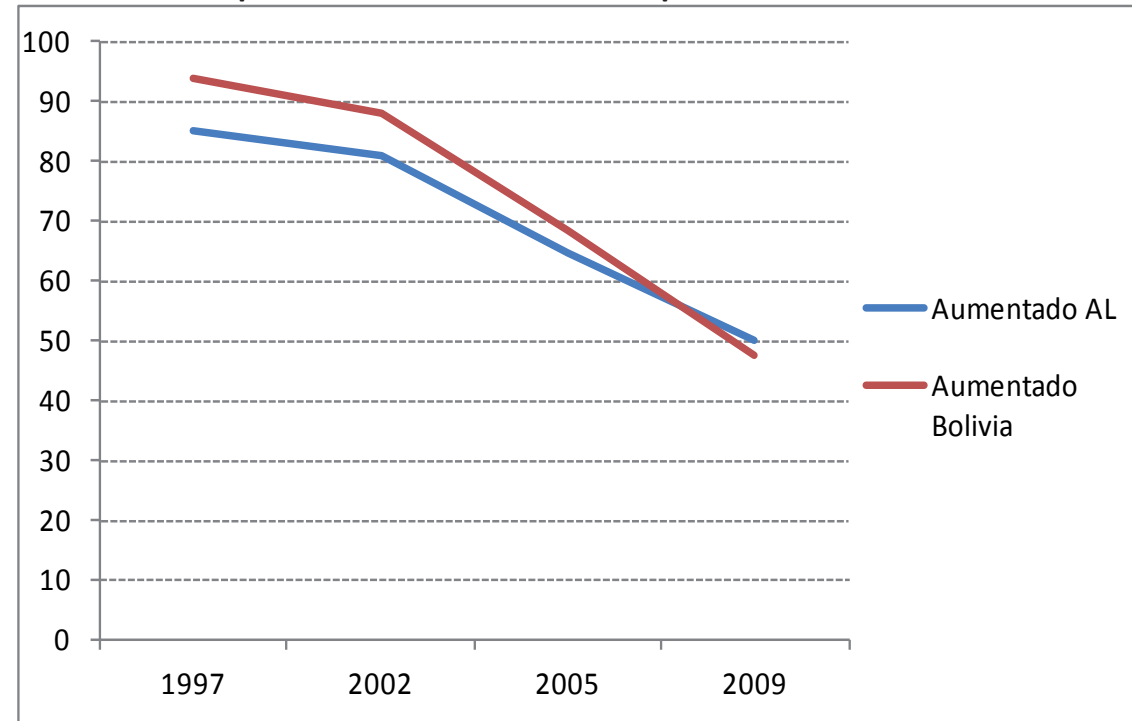

* La categoría "Aumentado" es la agregación de las respuestas de que la Corrupción ha aumentado mucho y que ha aumentado poco. Las otras posibilidades de respuesta para los encuestados fueron permanecido igual, disminuido poco y disminuido mucho.

Fuente: Corporación Latinobarómetro (1998), (2003), (2006) y (2010). 
En la primera gráfica destacan dos aspectos. El primero es que en todos los años examinados la confianza en los partidos políticos en Bolivia es inferior a la que se observa en toda la región latinoamericana, no obstante lo cual las trayectorias en ambos casos siguen la misma tendencia: descenso agudo a finales del siglo XX y principios del XXI e incremento paulatino posterior. El segundo es que la población boliviana tenía una aguda desconfianza hacia los partidos en 2002, año en que el MAS obtuvo el segundo lugar en las elecciones presidenciales. La confianza hacia los mismos mejoró notablemente (en casi 10 puntos porcentuales) durante el primer gobierno de Evo Morales, observándose que en 2009 la brecha entre las dos líneas de la gráfica es la más reducida de todo el período analizado (el rezago en confianza hacia partidos de Bolivia respecto a los de América Latina es el más bajo).

Estos datos indican que aunque los partidos jugaban con las reglas del sistema político que les permitía, vía pactos y coaliciones, acceder a un poder compartido que posibilitó una continuidad democrática inédita, no estaban preparados para el surgimiento de nuevos actores. Su poca legitimidad no sólo se mantuvo, sino que se incrementó a pesar de la reforma electoral de 1994. No obstante, el hecho de que se haya producido esta reforma es un indicador de que eran conscientes de las posibles consecuencias que este problema podía generar. El rediseño electoral no pudo modificar el comportamiento de los partidos ni pudo revertir la tendencia observada en la gráfica 1. En consecuencia, las instituciones no pudieron procesar el conflicto y se vieron impotentes ante un actor, el MAS, que jugaba adentro del sistema político pero lo golpeaba desde afuera. El incremento en la confianza a los partidos posterior a 2005 indica que efectivamente habían actores nuevos y sectores de la población que no estaban incluidos en el juego democrático de años previos.

La gráfica 2 nos muestra cambios más interesantes, puesto que la satisfacción con la democracia en Bolivia, al igual que la confianza en partidos, había estado sostenidamente por debajo de la observada para toda América Latina, alcanzando su punto más bajo en 2005, año del primer triunfo electoral de Morales. El contraste es notorio al ver que en los cuatro años de su primer gobierno, esta satisfacción aumentó casi en 30 puntos porcentuales, rebasando incluso la enorme mejoría que se observa en la región en el mismo lapso. La gráfica 3 confirma las tendencias de mejoría en la percepción con la democracia boliviana, ya que la opinión de que la corrupción "ha aumentado" no sólo disminuye en el período estudiado, sino que durante el primer gobierno del MAS ha revertido la brecha negativa en relación a toda Latinoamérica. Es notable que en 2009 menos de la mitad de los en- 
cuestados opine que la corrupción en Bolivia ha aumentado, cuando sólo doce años antes casi el 95\% percibía esto.

Las gráficas anteriores evidencian la muy baja satisfacción ciudadana con la democracia en Bolivia, con los partidos y con el desempeño público (señalado por la percepción sobre la corrupción) en los años previos a la presidencia de Evo Morales. Este desgaste guarda relación con la disminución del apoyo electoral hacia MNR, ADN y MIR, con la emergencia de nuevos partidos y con el posterior colapso del sistema de partidos, producido entre el autoexilio de Sánchez de Lozada y el primer triunfo del MAS (2003 a 2005). No obstante, existe literatura que señala que el derrumbe de los partidos y la transformación posterior del sistema político boliviano son consecuencia de los resultados adversos a nivel social que el modelo económico neoliberal habría generado (Seoane, 2006; Arriarán, 2007). Aunque hay estudios que muestran que los resultados económicos en ese país posteriores a la crisis de la deuda son en realidad satisfactorios (Laserna, 2004; Torrico, 2006), es necesario controlar la influencia de la percepción de la situación económico propia (que puede corresponder o no con el desempeño económico nacional) si queremos analizar los determinantes de la satisfacción con la democracia antes y después del primer triunfo de Morales. La comparación con América Latina realizada en las gráficas 1, 2 y 3 se mantiene a continuación.

\section{Cuadro 5. Influencia de la percepción política y económica sobre la satisfacción con la democracia}

\begin{tabular}{|l|c|c|c|c|}
\hline \multirow{4}{*}{} & \multicolumn{3}{|c|}{ Var. Dependiente: 1 Satisfecho; $0=$ No satisfecho } \\
\cline { 2 - 5 } & $\mathrm{AL}$ & Bolivia & $\mathrm{c}$ & \multicolumn{2}{c|}{ AL } & Bolivia \\
\cline { 2 - 5 } & $0.632^{* * *}$ & 1.438 & 1.048 & $0.251^{* * *}$ \\
\hline \multirow{2}{*}{ Constante } & $(0.086)$ & $(0.522)$ & $(0.106)$ & $(0.519)$ \\
\hline \multirow{2}{*}{$\begin{array}{l}\text { Situación } \\
\text { Económica familiar }\end{array}$} & $1.412^{* * *}$ & 1.136 & $1.208^{* * *}$ & $1.496^{* * *}$ \\
\cline { 2 - 5 } & $(0.020)$ & $(0.125)$ & $(0.022)$ & $(0.113)$ \\
\hline \multirow{2}{*}{$\begin{array}{l}\text { Comportamiento } \\
\text { de la Corrupción }\end{array}$} & $1.289^{* * *}$ & 0.957 & $1.314^{* * *}$ & 1.104 \\
\cline { 2 - 5 } & $(0.019)$ & $(0.139)$ & $(0.019)$ & $(0.099)$ \\
\hline \multirow{2}{*}{$\begin{array}{l}\text { Confianza en los } \\
\text { partidos }\end{array}$} & $0.671^{* * *}$ & $0.654^{* * *}$ & $0.617^{* * *}$ & $0.754^{* * *}$ \\
\cline { 2 - 5 } & $(0.017)$ & $(0.091)$ & $(0.021)$ & $(0.093)$ \\
\hline $\mathrm{N}$ & 17,713 & 738 & 15271 & 1,137 \\
\hline R2 Nagelkerke & 0.090 & 0.045 & 0.083 & 0.036 \\
\hline
\end{tabular}


Cuadro 5. Influencia de la percepción política y económica sobre la satisfacción con la democracia (continuación)

\begin{tabular}{|l|c|c|c|c|}
\hline \multirow{2}{*}{} & \multicolumn{3}{|c|}{ Var. Dependiente: 1 = Satisfecho; $0=$ No satisfecho } \\
\cline { 2 - 5 } & AL & Bolivia & AL & Bolivia \\
\cline { 2 - 5 } & $0.513^{* * *}$ & 0.948 & $0.382^{* * *}$ & 0.027 \\
\hline \multirow{2}{*}{ Constante } & $(0.111)$ & $(0.551)$ & $(0.103)$ & $(0.489)$ \\
\hline \multirow{2}{*}{$\begin{array}{l}\text { Situación } \\
\text { Económica familiar }\end{array}$} & $1.380^{* * *}$ & 1.040 & $1.557^{* * *}$ & $2.020^{* * *}$ \\
\cline { 2 - 5 } $\begin{array}{l}\text { Comportamiento } \\
\text { de la Corrupción }\end{array}$ & $(0.023)$ & $(0.120)$ & $(0.021)$ & $(0.107)$ \\
\cline { 2 - 5 } & $1.358^{* * *}$ & 1.124 & $1.524 * * *$ & $1.964 * * *$ \\
\hline $\begin{array}{l}\text { Confianza en los } \\
\text { partidos }\end{array}$ & $(0.019)$ & $(0.080)$ & $(0.018)$ & $(0.076)$ \\
\cline { 2 - 5 } & $0.584^{* * *}$ & $0.636 * * *$ & $0.617^{* * *}$ & 0.954 \\
\hline $\mathrm{N}$ & $(0.020)$ & $(0.099)$ & $(0.020)$ & $(0.083)$ \\
\hline R2 Nagelkerke & 16,745 & 1,086 & 17,055 & 1,121 \\
\hline
\end{tabular}

Situación económica familiar: 1=muy mala, 2=mala, 3=regular, 4=buena, y 5=muy buena.

Comportamiento de la corrupción: 1=ha aumentado mucho; 2 =ha aumentado poco; $3=$ =permanecido igual, 4=ha disminuido poco, y 5 =ha disminuido mucho.

Confianza en los partidos: 1=mucha; 2=algo; 3=poca, y 4=ninguna.

* Significancia al $10 \%$

** Significancia al $5 \%$

$* * *$ Significancia al $1 \%$

Todas las variables independientes en el modelo de regresión logística binaria del cuadro 5 resultan significativas en todos los años analizados para explicar la satisfacción con la democracia en América Latina. Además, el sentido de la relación es el lógicamente esperado, ya que la influencia de la percepción i) sobre la situación económica familiar es positiva (mejor percepción económica aumenta la satisfacción democrática), ii) sobre los cambios en la corrupción también es positiva (en la medida en que se piensa que la corrupción disminuye se incrementa la variable dependiente), y iii) sobre la confianza en los partidos es negativa (niveles menores de confianza disminuyen la probabilidad de que las personas expresen satisfacción con la democracia).

Los resultados en Bolivia son bastante más confusos que los observados en Latinoamérica. Aunque ninguna de las variables es significativa en todos los años analizados, la confianza en los partidos es la que muestra una influencia más sostenida, en especial en el período previo a la llegada de Morales a la presidencia, donde la amplia desconfianza vista en la Gráfica 1 explica buena parte de la poca satisfacción con la democracia observada en la gráfica 2. Es posible que el notable aumento de esta última entre 2005 
y 2009 haya provocado que en el último modelo de regresión la confianza en los partidos -que también subió, aunque en menor medida- deje de ser significativa. Este hallazgo confirma que la inconformidad social con el sistema de partidos en los noventa e inicios de este siglo estuvo relacionada con el desencanto con la democracia, lo que favoreció el crecimiento del MAS, como expresión del rechazo a la lógica de pactos. Este es hoy en día el único partido político medianamente organizado en Bolivia, por lo que se puede afirmar que prácticamente no existe sistema de partidos. Es posible que esto también esté afectando a que la variable "confianza en los partidos" no sea relevante en 2009, y que el aumento en confianza hacia los mismos sea en realidad confianza hacia el Movimiento al Socialismo.

La situación de la percepción sobre el comportamiento de la corrupción es opuesta a la de confianza en los partidos, ya que sólo en 2009 tiene relación significativa con la satisfacción con la democracia. Es difícil explicar por qué en un país la población puede juzgar a su sistema de gobierno independientemente de que perciba que el mencionado delito ha aumentado en el pasado inmediato. Una respuesta puede ser que a la gente le interesa el desempeño efectivo de gobierno con independencia de que los políticos estén obteniendo beneficios privados de las finanzas públicas. Al respecto, existe evidencia de que la corrupción en democracia no inhibe el crecimiento económico, por lo que podría ocurrir que convivan corrupción con buen desempeño (Drury et al., 2006). En los años analizados salvo 2009, los electores bolivianos se diferencian del resto de latinoamericanos por no vincular su satisfacción con la democracia y su percepción de la corrupción. Los altos niveles de pobreza imperantes pueden ocasionar este tipo de razonamiento pragmático (Herrera et al., 2007). ${ }^{13}$ En el mencionado año esto cambia y se observa una relación significativa entre las variables señaladas. De esta manera, la disminución en la percepción de que la corrupción aumentó influye en la mayor satisfacción con la democracia (gráficas 2 y 3 ).${ }^{14}$ Es posible nuevamente que en esto esté influyendo la popularidad del MAS.

13 Recordemos que Bolivia es el país más pobre de Sudamérica y que esta situación no ha cambiado en las últimas tres décadas.

No es intención analizar en detalle la relación entre percepción de la situación económica familiar y satisfacción con la democracia, ya que aquella es sólo un variable de control en el análisis. No obstante, resulta notorio que en algunos años no resulte significativa (en el resto de América Latina la relación es siempre significativa) y que sólo lo sea en años en los que se produjo una crisis económica. En 2002 aún se sentían los efectos de las crisis asiática y argentina, y en 2009 de la crisis financiera global. 
Sin duda existen más variables que explican la variable dependiente del cuadro 5. Nos hemos concentrado en aquellas que consideramos más importantes desde el punto de vista de las percepciones de los propios electores, puesto que aunque el desempeño objetivo de la economía y de las instituciones políticas son factores que importan, las creencias y valoraciones individuales por muy subjetivas que sean, son las que finalmente motivan a las personas no sólo a votar sino también a actuar.

\section{Prospectiva política}

¿Qué posibles escenarios se generarían con los cambios constitucionales para elegir al presidente dadas las percepciones observadas y el contexto político actual en Bolivia? Sin una segunda ronda congresal pero con segunda vuelta ciudadana se mantienen los incentivos de los partidos pequeños por participar en las elecciones a cambio de negociar después su apoyo. ${ }^{15}$ Aunque esta negociación tendría características diferentes ya que en el pasado el apoyo era visible a través del voto de los legisladores de esos partidos $\mathrm{y}$, bajo las nuevas reglas, no es obvio que los electores que votaron por un partido pequeño lo hagan por el que éste apoye en la segunda vuelta, el incentivo al multipartidismo persiste. Es así que en 2009 se presentaron ocho candidaturas presidenciales, igual número al que se observó en 2005. No obstante, debido a que la posibilidad de formar coaliciones de gobierno se reduce, ya que bajo las nuevas reglas no se necesita mayoría congresal para elegir al presidente en una eventual segunda ronda, lo más probable es que aunque persista el multipartidismo, paulatinamente el número de partidos participantes vaya reduciéndose. Esto implica que los electores irían concentrando sus votos en las candidaturas con mayores posibilidades de no quedarse en la primera vuelta. Además ya no estaría presente el incentivo para los votantes de impulsar al Congreso a su partido preferido, por pequeño que sea, para que sea parte de una posible coalición de gobierno. En este sentido habría mayor probabilidad de voto estratégico.

Por el momento el amplio respaldo electoral del MAS hace innecesaria la negociación con otros partidos, no solamente para mantener el poder si-

15 Más allá de que el Movimiento al Socialismo es el único partido relevante, existen muchos partidos de tamaño mediano y pequeño. 
no también para tomar decisiones, ${ }^{16}$ por lo que el gobierno de Evo Morales tiene la posibilidad de gobernar con eficacia lo que era muy difícil de alcanzar en gobiernos de coaliciones. ${ }^{17}$ No obstante, resulta natural pensar que en el futuro se volverá a la situación en la que ningún candidato obtendrá mayoría absoluta de votos en primera ronda, ${ }^{18}$ ¿qué podría ocurrir?

De no alcanzar ningún partido mayoría absoluta, necesariamente tendrá que negociar con partidos menores su apoyo para la segunda ronda, sin embargo, ello no necesariamente se tendría que traducir en coaliciones de gobierno, ya que como se dijo hay un elemento de incertidumbre respecto a si los votantes de los partidos pequeños se inclinarían por el partido más grande. Por otro lado, si no hay mayoría de votos para algún candidato, tampoco habrá mayoría en el congreso para algún partido, lo que daría lugar a una figura inédita en la política boliviana: la formación de gobiernos sin mayoría en el congreso. Ante esta situación la negociación permanente no sólo será necesaria, sino imprescindible para el funcionamiento democrático. Y ello es algo a lo que los políticos bolivianos no están muy acostumbrados.

En las últimas tres décadas, sólo en dos ocasiones el gobierno ha carecido de mayoría legislativa, y en ambos casos se vio forzado a adelantar elecciones. Hernán Siles (1982-1985), quien asumió el poder respaldado por una coalición de izquierda, sufrió luego la deserción de varios partidos ante la incapacidad de detener la crisis hiperinflacionaria y en vista de la imposibilidad de aprobar cualquier paquete de emergencia, ${ }^{19}$ no tuvo más opción que abandonar el poder. De forma similar, la crisis política de 2003-2005, derivada de la renuncia de Sánchez de Lozada, generó un

16 La Constitución de 2009 mantuvo el sistema electoral mixto con ajuste proporcional para la conformación de la Cámara de Diputados, lo que provoca que su conformación sea muy cercana a la distribución de votos para presidente.

Según datos de "El Barómetro de las Américas: Bolivia 2012", sólo el 25.7\% de los encuestados votaría en una elección presidencial por el candidato del MAS y el 44\% lo haría por algún partido de la oposición. El 30.3\% restante no iría a votar o anularía su voto. Aunque no se puede concluir de esto que es poco probable que el actual gobierno se mantenga en el poder, ya que la encuesta se realizó en marzo y abril de 2012, más de dos años y medio antes de las próximas elecciones, tampoco se puede descartar la posibilidad de que en 2014 se produzca una segunda vuelta y que, por tanto, el presidente electo no tenga mayoría en el Congreso.

19 Siles incluso recurrió a una huelga de hambre para que el Congreso aprobara sus propuestas, medida que fracasó y lo mostró políticamente aún más débil. 
gobierno coyuntural encabezado por Carlos Mesa quien a pesar de inicialmente señalar su intención de concluir el período de mandato hasta 2007, también se vio sin otra alternativa que renunciar. El MNR no le perdonó al ex vicepresidente haberse desmarcado de las decisiones del Ejecutivo en pleno estallido social y ningún partido tuvo el menor incentivo de respaldar al nuevo presidente, quien sólo duró en el cargo poco más de un año.

En las pocas ocasiones que el Ejecutivo ha visto entorpecida sus intenciones de aprobación legislativa, la reacción ha sido violenta. Inmediatamente se amenazaba a los partidos de la coalición con procesos judiciales por corrupción, o se removía a sus ministros y se empezaba a despedir a funcionarios pertenecientes a los mismos. Luego de un período tenso, la coalición se recomponía y el gobierno mantenía su mayoría legislativa y su posición dominante. En la presidencia de Evo Morales la reacción es distinta, ya que se moviliza a los sectores sociales y sindicatos afines al gobierno, y en ocasiones se cercan las instalaciones del Congreso hasta que éste apruebe las políticas de interés del Ejecutivo. Así se logró la aprobación del referéndum que luego ratificaría la nueva Constitución.

El escenario más probable ante la presencia de gobiernos sin mayoría legislativa sería la confrontación entre los partidos del oficialismo y la oposición, tanto en el Congreso como en las calles y carreteras. Ante este escenario lo peor que podría suceder es que se formaran dos bandos con fuerzas relativamente similares, ya que ello derivaría en crisis política en que se movilizarían sectores sociales afines a unos y otros. Frente a esta posibilidad, tal vez sea una buena noticia el multipartidismo, ya que atenúa la polarización. Aunque se prevé una paulatina concentración del voto del electorado en menos partidos y por lo tanto una eventual disminución del número de participantes en elecciones, es poco probable, dada la historia política y los múltiples clivajes existentes, que en Bolivia se genere un bipartidismo o surjan dos grandes coaliciones preelectorales. No obstante de ello, es claro que para tener estabilidad democrática a largo plazo tendrá que desarrollarse una cultura política de negociación, en la que los presidentes asuman que no siempre pueden ganar en el juego político. Dado el comportamiento político previo de las autoridades electas, el sistema electoral presidencial de la Constitución de 2009 generará estabilidad sólo si se mantiene la condición de que exista un partido en el gobierno con mayoría en el Congreso, la cual también era fundamental para el funcionamiento democrático en el lapso previo a la presidencia de Evo Morales. Ello revela uno de los límites del diseño institucional: la convivencia democrática en situaciones de ausencia de mayorías requiere de cambios culturales a fa- 
vor de una toma de decisiones incluyente. Es posible que el aumento en la satisfacción con la democracia de los electores facilite esta transformación.

\section{Conclusiones}

Este artículo muestra las dificultades de pensar las instituciones políticas únicamente como variables independientes en estudios de caso. Aunque es innegable que las instituciones influyen en el comportamiento de los actores políticos (como se muestra con el análisis de los efectos de la segunda ronda congresal en Bolivia), también son modificadas en función de los contextos políticos y al estudiar casos particulares éstos no pueden ser ignorados. A lo largo del documento se analizó cómo el sistema electoral presidencial prevaleciente entre 1980 y 2009 generó incentivos para la fragmentación del sistema de partidos y para la formación de coaliciones de gobierno. Pero también se mostró cómo el cambio en las preferencias electorales, los déficits de legitimidad de los partidos y los problemas de eficacia de los gobiernos llevaron a un cambio que de momento beneficia a la coalición ascendente establecida en el Movimiento al Socialismo.

El examen de las implicaciones de las nuevas reglas electorales nos indica, sin embargo, que algunos incentivos se mantienen (como los que conducen a un multipartidismo, aunque con características atenuadas) y que en el momento en que las preferencias de los electores le quiten el dominio político al MAS (lo cual es natural que suceda), lo más probable es que los futuros gobiernos se enfrenten a una situación en que no cuenten con mayoría legislativa, lo que podría amenazar la eficacia de las decisiones y la propia estabilidad política. ¿Esto podría generar una nueva pérdida de legitimidad de los partidos? Todo dependerá de cómo se gestione el conflicto, pero una salida podría ser el diseño de instituciones que incentiven la negociación o desconcentren las decisiones, por ejemplo, a través del impulso a las autonomías departamentales, lo que daría mayor poder al ámbito local en detrimento de las autoridades nacionales.

Sin duda es una buena noticia que los electores sientan mayor satisfacción democrática, perciban menor corrupción y confíen más en los partidos. Esto evidencia que con el gobierno de Evo Morales un amplio sector de la población que se sentía excluido o estaba inconforme con el funcionamiento de la democracia ya encuentra que ella puede resolver sus problemas o que al menos se le toma en cuenta. Esto constituye una base importante para el sostenimiento de las libertades y los derechos que los actores políticos no deberían dilapidar. La toma de decisiones negociadas 
ante una eventual disminución del margen de victoria de cualquier partido que llegue al gobierno, sería el siguiente paso para la consolidación democrática pero ello implicará un cambio en la lógica política imperante por más de tres décadas.

\section{Bibliografía}

Arriarán, Samuel, 2007, La derrota del neoliberalismo en Bolivia, México, Torres Asociados.

Arrow, Kenneth J., 1963, Social choice and individual values, New Haven, Yale University Press.

Buquet, Daniel, 2007, “Entre la legitimidad y la eficacia: Reformas electorales en América del Sur", Revista Uruguaya de Ciencia Politica,16, pp. 35-49.

Corporación Latinobarómetro, 1998, 2003, 2006 y 2010, Encuesta Latinobarómetro, Santiago de Chile, bases de datos disponible por Internet en: $\underline{w w w . l a t i n o b a-}$ rometro.org.

Cox, Gary y Scott Morgenstern, 2002, “Epilogue: Latin America's Reactive Assemblies and Proactive Presidents", en Scott Morgenstern y Benito Nacif (eds.), Legislative Politics in Latin America, Cambridge, Cambridge University Press.

Cox, Gary W.,1997, Making Votes Count, Nueva York, Cambridge University Press.

Drury, A.C., J. Krieckhaus y M. Lusztig, 2006, “Corruption, democracy, and economic growth", International Political Science Review, vol. 27, 2, pp. 121-136.

Duverger, Maurice, 1961, Los partidos políticos, México, Fondo de Cultura Económica.

Herrera, Javier, Mireille Razafindrakoto y François Roubaud, 2007, “Governance, democracy and poverty reduction: lessons drawn from household surveys in sub-Saharan Africa and Latin America", International Statistical Review, vol. 75, 1, pp. 70-95.

Laserna, Roberto, 2004, “Bolivia: la crisis de octubre y el fracaso del ch'enko. Una visión desde la economía política”, en Anuario Social y Político de América Latina y el Caribe Núm. 7, Caracas, Flacso/UNESCO/Nueva Sociedad, pp 52-68.

Linz, Juan, 1994, “Democracia presidencial o parlamentaria ¿Qué diferencia implica?" en Juan Linz y Arturo Valenzuela (comps.) La crisis del presidencialismo, Madrid, Alianza, pp. 25-143. 
Mayorga, René Antonio, 2000, “Presidencialismo parlamentarizado y gobiernos de coalición en Bolivia", en Jorge Lanzaro (comp.), Tipos de presidencialismo y coaliciones políticas en América Latina, Buenos Aires, CLACSO.

Negretto, Gabriel, 2006, “Choosing How to Choose Presidents: Parties, Military Rulers, and Presidential Elections in Latin America", The Journal of Politics, vol. 68, 2, pp. 421-433.

Nohlen, Dieter (ed.), 2005, Elections in the Americas: a data handbook, Vol. 2, Oxford, Oxford University Press.

OEP, 2012, Estadísticas Electorales, La Paz, Órgano Electoral Plurinacional, disponible en http:/ / www.oep.org.bo/proces_electoral/procesos1985_1995.aspx

Olson, Mancur, 1991, La lógica de la acción colectiva, México, Limusa, Grupo Noriega Editores.

Proyecto de Opinión Pública de América Latina (LAPOP), 2012, El Barómetro de las Américas, base de datos disponible por Internet en: www.LapopSurveys.org.

Rae, Douglas W., 1967, The political consequences of electoral laws, New Haven, Yale University Press.

Reynolds, Andrew, Ben Reilly y Andrew Ellis, 2006, Diseño de sistemas electorales. El nuevo manual de IDEA Internacional, México, IDEA Internacional e Instituto Federal Electoral y Tribunal Electoral del Poder Judicial de la Federación.

Riker, William H., 1982, Liberalism against populism, Illinois, Estados Unidos, Waveland.

Seoane, José, 2006, “Movimientos sociales y recursos naturales en América Latina: resistencias al neoliberalismo, configuración de alternativas" Sociedade e Estado, vol. 21, 1, Brasilia, pp. 85-107

Shugart, Matthew S. y Martin P. Wattenberg, 2001, Mixed-Member Electoral Systems. The Best of Both Worlds?, Oxford, Oxford University Press.

Taagepera, Rein y Matthew S. Shugart, 1989, Seats and Votes, New Haven, Yale University Press.

Torrico, Mario, 2006, “Qué ocurrió realmente en Bolivia?”, Perfiles Latinoamericanos, núm 26, México, pp. 231-261.

Recibido el 13 de junio de 2013 Aceptado el 6 de noviembre de 2013 